\title{
On the Optimum Number of Fourier Descriptors for Closed Boundary Retrieval
}

\author{
Ramamurthy Suri ${ }^{a}$, Gopikrishna Pulluri ${ }^{a}$ and Keerthana Nakka ${ }^{b}$ \\ ${ }^{a}$ Department of Humanities and Sciences, Gokaraju Rangaraju Institute of Engineering and \\ Technology, Hyderabad-500 090, India \\ ${ }^{b}$ ECE, Advanced Academic Center, Gokaraju Rangaraju Institute of Engineering and Technology, \\ Hyderabad-500 090, India \\ e-mail: suriramamurthy7@gmail.com
}

\begin{abstract}
In the post segmentation scenario, when objects in the scene have been extracted, the focus shifts to object identification. This can be achieved through shape or texture. Finding the object boundary has been a reliable means of shape description. Among the mathematical approximation techniques for shape analysis, Fourier descriptors have proven to approximate closed boundaries of objects quite well, albeit with some limitations. A statistical thresholding technique to restrict the number of descriptors for a reasonably good approximation of the target shape is explored and tested on some medical images. Encouraging results were obtained particularly when segmentation in the pre processing stage was effectively carried out.
\end{abstract}

Keywords: Boundary description, Error metrics, Fourier descriptors, Object recognition, Parameter optimization, Shape matching

\section{Introduction}

Humans are bestowed with an instinct to recognize objects and patterns that are associated in a scene even when the context is not very clear. Processing, analysis and understanding a scene almost happens seamlessly. It is however quite possible that human recognition may fail particularly if what to expect in an image is unknown. One area where human vision may fail is in the study of tumours where one may not have the knowledge of anatomy or microbiology. While the cognitive power of the human is very difficult to replicate through the machine, a certain class of problems in pattern recognition and scene analysis have been successfully tried even when the object of interest has many features. This specialized area of study has given scope to researchers to design various domain specific algorithms for complex problems. In the realm of computer vision, describing shape has opened new vistas of research. It is obvious that one cannot ideally define what shape means and what one has to look for in shape. In the external point of view, shape could be described through object skeleton or region boundary or some topological features, while from the interior, it could be described through colour and texture. This study involves object recognition though region boundary representation using Fourier descriptors. While this area has been very well researched, the attempt in this work is to put a cap on the number of descriptors 
for reasonable recognition instead of using too many of them. A novel statistical thresholding technique is developed to achieve the best approximation through the proposed approach.

[1. 2have dealt with the concept of Fourier descriptors in the study of shapes and effective retrieval. 3] have applied Fourier descriptors in the classification of blood smears. 4, 5. have dealt with shape analysis using Fourier descriptors for 2-D closed curves. [6] is one of the earliest papers that has contributed to the line of research on Fourier descriptors. [7, 8, have used Fourier descriptors for character recognition problems. [9] have applied Fourier descriptors for leaf classification problems.

[10, 11, 12, 13, 14 have addressed the problem of leaf classification using Fourier descriptors and other shape features. [15] have implemented an algorithm for the edge detection of $\mathrm{X}$ - ray images. [16, 17, 18] have applied spectral methods in the shape analysis of skin lesions. 19] reviews various metrics for shape evaluation. 20] have estimated the PSNR and MSE on lossless compressed digital images. [21] survey supervised edge detection evaluation methods. [22, 23, 24, 25] are classic text books for the mathematical aspects of approximations using Fourier descriptors. [26] was conceived as a novel parametrization scheme in the spatial domain for modelling open curves with complex shapes. 27, 28, were resources used by the authors for assimilation of techniques in bio-medical signal processing, though not directly in line with the current application.

The paper is organized as follows: The representation of object boundary both in the spatial and spectral domains is discussed in Section 2 Shape signatures which form the backbone for applying Fourier descriptors are discussed in Section 3 with an emphasis on the complex shape signature. The mathematical aspects of Fourier descriptors based on the complex shape signature is presented in Section 4 The algorithm that puts a cap on the number of Fourier descriptors for a fair representation of object shape is proposed in Section 5 and tested on an image database consisting of some X-ray images and skin lesions. Well established error metrics have been used to justify the approximation technique We have incorporated the Mean square error (MSE) and Peak signal to noise ratio (PSNR) in the analysis. Experimental results and discussion are incorporated in Section 6 Section 7 deals with conclusion and recommendations of the study. The iterative procedure to optimize the parameter that limits the number of FDs for an fair approximation of the target shape has been successfully tested on a database of medical images. Section 8 highlights the future scope of study. The promising results in this study has motivated the authors to focus on efficient segmentation of medical images, tumour lesions in particular, for better approximation of the target boundaries. Novel approximation techniques, both in the spatial and spectral domains can be taken up in tandem and evaluated against some error metrics for different scenarios.

\section{Boundary Representation}

Broadly, there are two approaches to shape boundary description: spatial and spectral. Spatial descriptors are based on region boundary include boundary length, curvature, bending energy, chord distribution, to mention a few. Since digital images are stored in discrete data structures, they are highly sensitive to scale change. Most spatial shape descriptors suffer from this defect. Descriptors defined in the frequency domain deal with the issues of noise and scale more efficiently. Fourier descriptors encode the boundary in the spectral domain. They can be designed to be independent of scaling, translation, or rotation because shape is that aspect of the object that is invariant of the above.

\section{Shape Signatures}

A shape signature function is a one-dimensional function defined on the pixel information of the region boundary with the requirement that it is periodic. An obvious choice is to make the shape signature function a complex function. This permits one to have a Fourier series for the function. Shape signatures have been defined in several ways. To visualize how one determines these descriptors, we digitize the object boundary thereby finding the coordinates $(\mathrm{x}, \mathrm{y})$ of each boundary pixel. Imagine a $k$ - point digital 
boundary in the $X Y$ - plane. Assuming that these $k$ points are ordered, we start from an arbitrary point $\left(x_{0}, y_{0}\right)$. Subsequent points $\left.\left(x_{1}, y_{1}\right),\left(x_{2}, y_{2}\right), \ldots,\left(x(k-1), y_{(} k-1\right)\right)$ are encountered as one traverses in, say, the anti clockwise direction. Among the several shape signature functions that have been defined, the Complex shape function, Centroid distance function, Curvature function, Cumulative angle function are some. We focus on the complex shape function which we employ for the problems in this study. In this case, each point can be represented as a complex function $z(k)=x(k)+i y(k)$. Each point can be represented as a complex function where $k$ is a measurement related to the curve. To ensure invariance under translation, the mean of the shape is subtracted.

$$
z(k)=\left(x(k)-x_{0}\right)+i\left(y(k)-y_{0}\right)
$$

This representation reduces a 2 -dimensional problem to that of a single dimension.

\section{Fourier Descriptors}

One can determine Fourier descriptors by applying the discrete Fourier transform (DFT) on the shape signature function. The DFT of this function generates Fourier coefficients $a_{u}$ determined by

$$
a_{u}=\sum_{k=0}^{K-1} z(k) \exp (-i 2 \pi k u / K)
$$

where $u=0,1,2, \ldots,(K-1)$.

The complete coefficients $a_{k}$ are called the Fourier descriptors of the boundary. The inverse discrete Fourier transform (IDFT) of these coefficients restores $z(k)$.

$$
z(k)=\frac{1}{K} \sum_{u=0}^{K-1} a_{u} \exp (2 \pi i u k / K)
$$

This result is an analysis technique credited to [6, 7]. Fourier descriptors are used as a symbolic representation of shape for subsequent representation. With the aid of a few descriptors one can visualize the gross shape and by considering more descriptors, finer details of the shape reveal themselves. The number of descriptors to be considered will depend on the amount of detail one is looking for.

For applications in medical imaging, one may need to compute Fourier descriptors of high order to capture fine details that is so essential for diagnosis. It is therefore necessary not to invest too much on computing lower order descriptors. We propose a technique that determines the optimum order of the Fourier descriptor based on minimizing a parameter, for a fair approximation to the target shape.

\section{Proposed Method}

We propose an iterative technique where the target shape statistics are evaluated against those of a set of sample shapes that satisfies an error criterion. Let $\mu$ and $\sigma$ be the mean and standard deviation of the target shape. Now we generate a sample shape by using a low order descriptor, say using order 5 . We determine the mean $\mu_{1}$ of this shape. A tolerance say $\varepsilon=5 \times 10^{-4}$ is fixed to compare the shapes of the target and the sample. We compute the ratio $\left|\frac{\mu-\mu_{1}}{x \sigma}\right|$. If $\left|\frac{\mu-\mu_{1}}{x \sigma}\right| \leq \varepsilon$, the sample shape is a good enough approximation to the target for an appropriate $x$. The strategy is to find the smallest value of $\mathrm{x}$, the model parameter, in the range $1.4 \leq x \leq 3$. Thus, we optimize $x$ by which a matching occurs. If $\left|\frac{\mu-\mu_{1}}{x \sigma}\right|>\varepsilon$, the first sample is discarded and we build a new sample shape with, say, 10 descriptors, and so on till a suitable match between the new sample and the target is found. This is the iterative approach to shape matching we wish to explore. 


\subsection{Proposed Algorithm}

1. Get target shape, compute the mean $\mu$ and standard deviation $\sigma$ of the shape.

2. Set error tolerance $\varepsilon=5 \times 10^{-4}$, a parameter $x$ in the range $1.4 \leq x \leq 3$ and initial order of shape descriptor $i=k$ ( $k$ constant).

3. Initialize sample shape $S_{i}$ with $i$ descriptors. Compute the mean $\mu_{i}$ of the sample shape. Designate $y=\left|\frac{\mu-\mu_{1}}{\varepsilon x \sigma}\right|$.

4. If $y \leq 1, S_{i}$ is the optimum approximation at this resolution $(k)$ and Stop.

5. Else, set $i=i+5$ and go to 3 .

\subsection{Error Metrics}

The quality of the edges extracted from an image is assessed through error metrics like MSE, PSNR and SSIM. Two metrics we use in our analysis are MSE and PSNR. The Mean square error (MSE) is a weighted function of deviations in images, or square difference between compared images. Formally it is defined as

$$
M S E=\frac{1}{M N} \sum_{i=1}^{M} \sum_{j=1}^{N}\left(I_{1}(i, j)-I_{2}(i, j)\right)^{2}
$$

Where $M$ and $N$ relate to image size, $I_{1}$ and $I_{2}$ refer to image locations. Smaller the MSE, closer the predicted and targeted images are.

The Peak signal noise ratio (PSNR) is an index of level of losses or signal integrity. It is defined as

$$
P S N R=10 \log \left(\frac{\max (I)^{2}}{M S E}\right)
$$

The definition of PSNR suggests that a low MSE results in a higher PSNR value. And the higher PSNR value, is an index of high image quality.

\section{Experimental Results and Discussion}

In all, fifteen medical images have been considered for the study. These images have been subjected to pre- processing, some needing segmentation, some needing simple thresholding and others needing adaptive thresholding techniques, to extract the desired object of interest. Then the object boundary was extracted. While some objects have simple boundaries, some have boundaries with varying complexities. These boundaries have been approximated using the proposed approach. We chose to limit the range $1.4 \leq x \leq 3.0$ for the parameter $x$. The determination of the optimal value of $x$ with respect to the hand $\mathrm{X}$-ray image evolved through the algorithm is explained in Table 1

Figure 1 shows a grayscale hand $\mathrm{X}$ - ray image [29], its binarization, boundary extraction and approximation using Fourier descriptors at various levels of resolution. This particular case boundary has 911 points, which theoretically needs as many Fourier descriptors for exact reproduction. We however have achieved a great amount of economy using just 395 descriptors with the parameter value $x=2.6$ for a fair approximation using the proposed algorithmic. Fugure 2 shows a gangrene RGB image [30] which was segmented, had the boundary extracted and approximated using the complex shape signature. Table 1 shows the determination of the optimization parameter $x$ for the hand X-ray image. The best approximation in this case required 270 descriptors while the boundary has 562 points. Figure 3 gives 


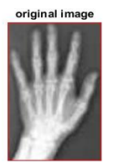

(a)

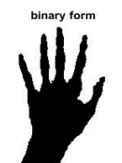

(b)

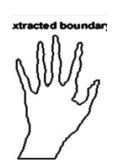

(c)

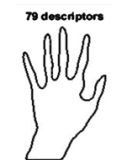

(d)

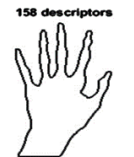

(e)

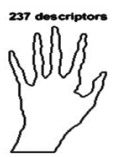

(f)

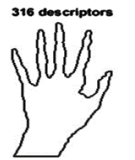

(g)

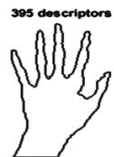

(h)

Figure 1. (a) A hand X - ray image, (b) its binarization, (c) object boundary (d-h) boundary approximation using 79, 158, 237, 316 and 395 descriptors.

Table 1. Determination of the optimal value of parameter $x$.

\begin{tabular}{|c|c|c|c|c|c|c|c|c|c|}
\hline$x$ & 1.4 & 1.6 & 1.8 & 2.0 & 2.2 & 2.4 & 2.6 & 2.8 & 3.0 \\
\hline \hline$y$ & $1.56 \times 10^{-4}$ & $1.37 \times 10^{-4}$ & $1.22 \times 10^{-4}$ & $1.09 \times 10^{-4}$ & $9.98 \times 10^{-5}$ & $9.15 \times 10^{-5}$ & $8.45 \times 10^{-5}$ & $1.56 \times 10^{-4}$ & $1.46 \times 10^{-4}$ \\
\hline
\end{tabular}

a visual picture of how the optimization was achieved for the images in Figures 11 and 2 Figure 4 is a database of six X-ray images [29, 31, 32, 33] and nine skin lesion images [30, 34, 35] which forms the subject to our study. Figure 5 displays the approximation of the object boundaries of the six X-ray images. Figure 6 displays the approximation of the object boundaries of the nine skin lesion images. Table 2 provides the summary of the study of the X-ray image database which includes the optimization parameter $x$, the number of points on the target boundary, the number of Fourier descriptors used for optimal boundary representation, the MSE and PSNR in each case. Table 3 provides similar information for the skin lesion image database. Low MSE and fairly high PSNR values suggest the robustness of the proposed approach.

The evolution of the parameter $x$ for the hand $\mathrm{X}$ - ray image obtained through the algorithm is given in the Table 1

The MSE and PSNR for this case are determined as $7.17 \times 10^{-4}$ and 79.57673 respectively, both emphasising the quality of the approximation.

The MSE and PSNR for this case are determined as $1.01 \times 10^{-4}$ and 79.5767 respectively.

A minimum is found at $x=2.6$ for which the first best approximation is obtained consuming 395 descriptors for case (a). A minimum is found at $x=2.4$ for which the first best approximation is obtained consuming 270 descriptors for case (b).

\section{Conclusion}

Fourier descriptors encode the shape of a boundary in the frequency domain. If one uses as many descriptors as the number of boundary points, exact reproduction of shape will be achieved. This however will not be required. One can limit the number of descriptors using a stopping rule. We have suggested a statistical thresholding algorithm to determine the optimum number of descriptors for a fair boundary approximation. The technique has been tried on a few medical images, which come with varying features. The study was worthwhile as the results were satisfactory. Two standard error metrics, the MSE and PSNR have been used as testing tools to test the robustness of the boundary approximation. The 


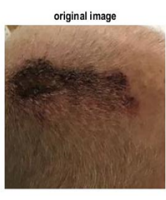

(a)

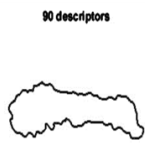

(d)

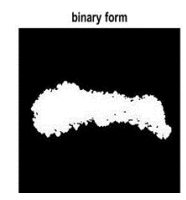

(b)

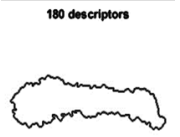

(e)

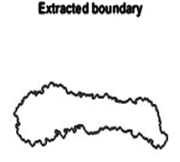

(c)

Figure 2. (a) A gangrene colour image (b) binarization after segmentation (c-f) boundary approximation using 90, 180 and 270 descriptors.

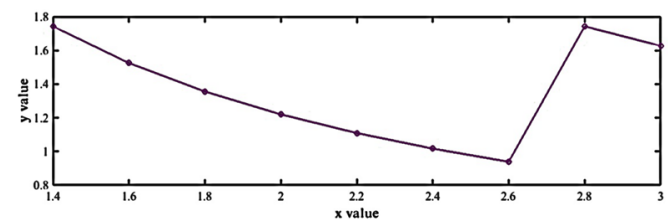

(a)

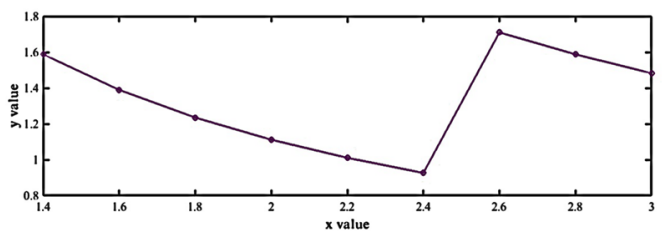

(b)

Figure 3. A graphic display of the optimization parameter $x$ for the two cases in (a) Figure 1 and (b) Figure 2 
Table 2. Analysis of approximations of X-ray images in the database.

\begin{tabular}{|c|c|c|c|c|c|}
\hline Image & $\begin{array}{c}\text { Optimal } \\
\text { value of } \\
\text { parameter } \\
x\end{array}$ & $\begin{array}{c}\text { Number of } \\
\text { boundary } \\
\text { points }\end{array}$ & $\begin{array}{c}\text { No. of } \\
\text { descriptors } \\
\text { used for best } \\
\text { approxima- } \\
\text { tion }\end{array}$ & MSE & PSNR \\
\hline & 2.6 & 911 & 395 & 0.0007166 & 79.5767 \\
\hline & 2.6 & 2105 & 1010 & 0.0011655 & 77.4653 \\
\hline & 1.4 & 1001 & 450 & 0.0004973 & 81.1661 \\
\hline & 3.0 & 674 & 340 & 0.0019030 & 75.3363 \\
\hline & 3.0 & 1998 & 865 & 0.0004113 & 81.9887 \\
\hline & 1.8 & 1037 & 450 & 0.0006480 & 80.0147 \\
\hline
\end{tabular}


Table 3. Analysis of approximations of skin lesion images in the database.

\begin{tabular}{|c|c|c|c|c|c|}
\hline Image & $\begin{array}{c}\text { Optimal } \\
\text { value of } \\
\text { parameter } \\
x\end{array}$ & $\begin{array}{c}\text { Number of } \\
\text { boundary } \\
\text { points }\end{array}$ & $\begin{array}{c}\text { No. of } \\
\text { descriptors } \\
\text { used for best } \\
\text { approxima- } \\
\text { tion }\end{array}$ & MSE & PSNR \\
\hline & 2.4 & 562 & 270 & 0.0010110 & 78.0828 \\
\hline & 1.6 & 836 & 410 & 0.0002038 & 85.0477 \\
\hline & 2.6 & 1202 & 550 & 0.0115096 & 67.5262 \\
\hline & 2.0 & 1486 & 715 & 0.0004402 & 81.6947 \\
\hline & 2.8 & 1078 & 440 & 0.0003974 & 82.1376 \\
\hline & 2.6 & 1133 & 525 & 0.0004136 & 81.9678 \\
\hline & 2.0 & 1362 & 605 & 0.0000016 & 81.1916 \\
\hline & 2.8 & 1219 & 610 & 0.0006255 & 80.1675 \\
\hline & 2.8 & 1530 & 660 & 0.0011191 & 77.6418 \\
\hline
\end{tabular}




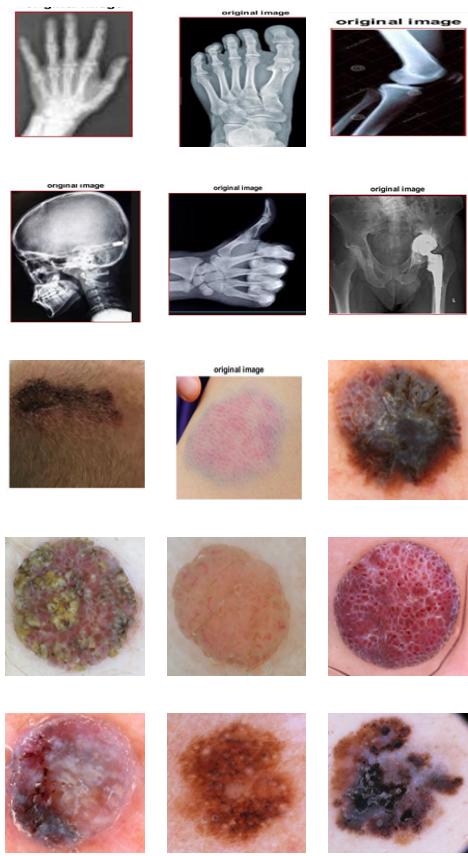

Figure 4. Database of some medical images: $\mathrm{X}$ - ray images (Top two rows) and Skin lesion images (Last three rows).

challenges that remain to be tackled are those images which are embedded in noise and other segmentation isuues. Cases where the PSNR values are relatively low need better pre-processing.

\section{Scope for Future Study}

Medical images, particularly those concerning tumor lesions, requore sophisticated segmentation algorithms. We propose to focus on effective segmantation procedures to extract objects of interest from the scene. Once segmented, we wish to apply spatial and spectral methods to reproduce the object boundary as faithfully as possible using efficient algorithms. This by itself is a non-trivial problem. Once the boundary is extracted and made amenable to mathematical manipulations, we propose to estimate image statistics like perimeter, area and other shape characteristics using mathematical ans statistical approaches. 

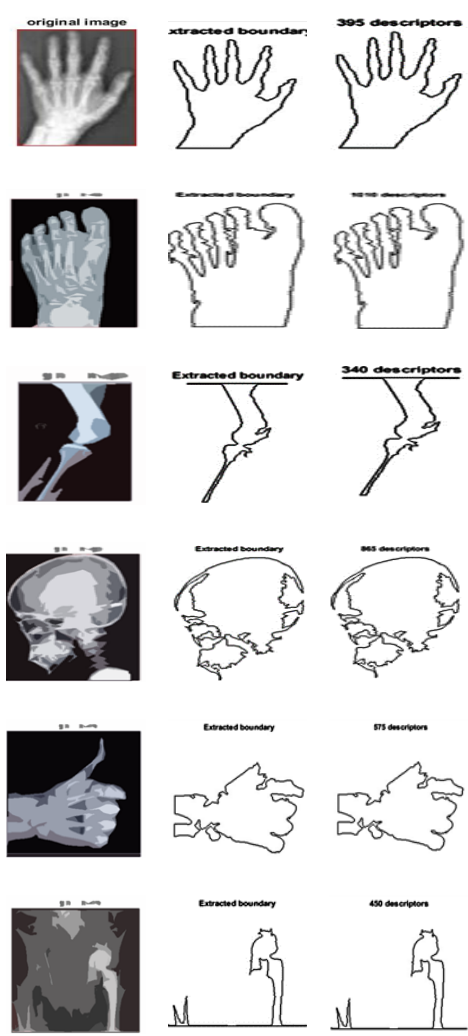

Figure 5. Results of processing X -ray images (1st column) original image, (2nd column) target boundary and (3rd column) boundary approximation. 

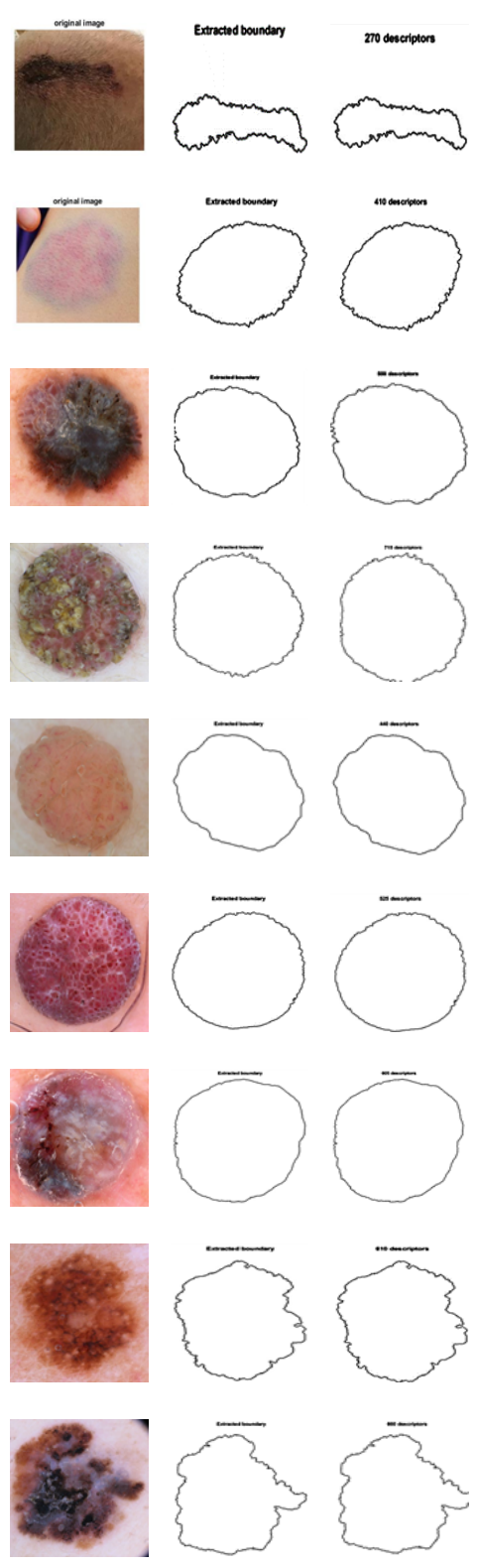

Figure 6. Results of processing X -ray images (1st column) original image, (2nd column) target boundary and (3rd column) boundary approximation. 


\section{References}

[1] Eric Persoon and King-Sun Fu. Shape Discrimination Using Fourier Descriptors. IEEE Transactions on Systems, Man, and Cybernetics, 7(3):170-179, 1977.

[2] Dengsheng Zhang and Guojun Lu. A comparative study of Fourier descriptors for shape representation and retrieval. In Fifth Asian Conference on Computer Vision (ACCV2002), pages 646-651, 2002.

[3] Rimma Tomakova, Vitaliy Komkov, Evgeniy Emelianov, and Maksim Tomakov. The Use of Fourier Descriptors for the Classification and Analysis of Peripheral Blood Smears Image. Applied Mathematics, 8(11):1563-1571, 2017.

[4] Adam Krzyzak, Siu Yun Leung, and Ching Suen. Fourier Descriptors of Two Dimensional ShapesReconstruction and Accuracy. In IAPR Workshop on Computer Visionn: Special Hardware and Industrial Applications, pages 199-202, Tokyo, 1988.

[5] Charles T. Zahn and Ralph Z. Roskies. Fourier Descriptors for Plane Closed Curves. IEEE Transactions on Computers, C-21(3):269-281, 1972.

[6] L. Cosgriff. Identification of Shape. Technical Report 820-11, Ohio State University Research Foundation, 1960.

[7] E.L. Brill. Character recognition via Fourier Descriptors. In Qualitative pattern recognition through image shaping, pages 1-10, LA, California, 1968.

[8] G. H. Granlund. Fourier Preprocessing for Hand Print Character Recognition. IEEE Transactions on Computers, C-21(2):195-201, 1972.

[9] Z. Wang, Z. Chi, and D. Feng. Shape based leaf image retrieval. IEE Proceedings - Vision, Image and Signal Processing, 150(1):34-43, 2003.

[10] Yeni Herdiyeni, Elvira Nurfadhilah, Ervizal A.M. Zuhud, Ellyn K. Damayanti, Kohei Arai, and Hiroshi Okumura. A Computer Aided System for Tropical Leaf Medicinal Plant Identification. International Journal on Advanced Science, Engineering and Information Technology, 3(1):23, 2013.

[11] Abdul Kadir, Lukito Edi Nugroho, Adhi Susanto, and Paulus Insap Santosa. Foliage Plant Retrieval using Polar Fourier Transform, Color Moments and Vein Features. Signal 6 Image Processing : An International Journal, 2(3):1-13, 2011.

[12] Abdul Kadir. Leaf Identification Using Fourier Descriptors and Other Shape Features. Gate to Computer Vision and Pattern Recognition, 1(1):3-7, 2015.

[13] Maliheh Shabanzade, Morteza Zahedi, and Seyyed Amin Aghvami. Combination of Local Descriptors and Global Features for Leaf Recognition. Signal 8 Image Processing : An International Journal, 2(3):23-31, 2011.

[14] Tomáš Suk, Jan Flusser, and Petr Novotný. Comparison of Leaf Recognition by Moments and Fourier Descriptors. In Computer Analysis of Images and Patterns, pages 221-228. Springer, 2013.

[15] Anil K. Bharodiya and Atul M. Gonsai. An improved edge detection algorithm for X-Ray images based on the statistical range. Heliyon, 5(10):e02743, 2019.

[16] Enes Albay and Mustafa Kamaşak. Skin lesion classification using fourier descriptors of lesion borders. In 2015 Medical Technologies National Conference, pages 1-4, 2015.

[17] Jian Liu, Wantao Wang, Jie Chen, Guozhong Sun, and Alan Yang. Classification and Research of Skin Lesions Based on Machine Learning. Computers, Materials \& Continua, 62(3):1187-1200, 2020.

[18] K.M. Clawson, P.J. Morrow, B.W. Scotney, D.J. McKenna, and O.M. Dolan. Determination of Optimal Axes for Skin Lesion Asymmetry Quantification. In 2007 IEEE International Conference on Image Processing, volume 2, pages II - 453-II - 456, 2007.

[19] Diana Sadykova and Alex Pappachen James. Quality assessment metrics for edge detection and edge-aware filtering: A tutorial review. 2017 International Conference on Advances in Computing, Communications and Informatics (ICACCI), pages 2366-2369, 2017.

[20] M. Manju, P. Abarna, U. Akila, and S. Yamin. Peak Signal to Noise Ratio \&Mean Square Error cal- 
culation for various Images using the lossless Image compression in CCSDS algorithm. International Journal of Pure and Applied Mathematics, 119(12):14471-14477, 2018.

[21] Baptiste Magnier, Hasan Abdulrahman, and Philippe Montesinos. A Review of Supervised Edge Detection Evaluation Methods and an Objective Comparison of Filtering Gradient Computations Using Hysteresis Thresholds. Journal of Imaging, 4(6):74, 2018.

[22] Rafael C. Gonzalez and Richard Eugene Woods. Digital Image Processing. Prentice Hall, 2008.

[23] Anil K. Jain. Fundamentals of Digital Image Processing. Prentice Hall, 1989.

[24] William K. Pratt. Introduction to Digital Image Processing. CRC Press, 2013.

[25] Luciano da Fontoura Costa and Roberto Marcond Cesar Jr. Shape Analysis and Classification: Theory and Practice. CRC Press, 2010.

[26] S N N Pandit, S Ramamurthy, and B Krishna Gandhi. Curve fitting when the curve may not be a function. Journal of Interdisciplinary Mathematics, 9(3):551-568, 2006.

[27] K. Padmavathi and K. Sri Ramakrishna. Classification of ECG Signal during Atrial Fibrillation Using Autoregressive Modeling. Procedia Computer Science, 46:53-59, 2015.

[28] K Swaraja, K Meenakshi, and Padmavathi Kora. An optimized blind dual medical image watermarking framework for tamper localization and content authentication in secured telemedicine. Biomed Signal Process Control, 55:101665, 2020.

[29] http://unisci24.com/

[30] https://www.walesonline.co.uk/news/wales-news/cyclist-suffered-blood-clot-brain-12766353.

[31] https://radiopaedia.org/

[32] https://www.dreamstime.com/

[33] https://www.futurity.org/x-rays-perovskite-nanocrystals-1869732/

[34] https://www.onhealth.com/content/1/bruises_causes_treatments_colors

[35] https://biomedicalimaging.org/2017/challenges// 